\title{
How "True Bitcoiners" Work on Reddit to Maintain Bitcoin
}

Megan L. Knittel

Michigan State University

East Lansing, MI, USA

knittel2@msu.edu

\author{
Rick Wash \\ Michigan State University \\ East Lansing, MI, USA \\ wash@msu.edu
}

\section{ABSTRACT}

After the meteoric rise in price, and subsequent public interest, of the cryptocurrency Bitcoin, a developing body of work has begun examining its impact on society. In recent months, as Bitcoin's price has rapidly declined, uncertainty and distrust have begun to overshadow early enthusiasm. In this late-breaking work, we investigated one of the largest and most important Bitcoin online communities, the r/Bitcoin Reddit forum. A vocal subgroup of users identify themselves as "true Bitcoiners", and justify their continued devotion to Bitcoin. These subreddit participants explained and justified their trust in Bitcoin in three primary ways: identifying characteristics of beneficial versus harmful Bitcoin users, diminishing the importance of problems, and describing themselves as loyal to Bitcoin over time.

\section{KEYWORDS}

Bitcoin; online community; trust

\section{INTRODUCTION}

In 2009, the cryptocurrency Bitcoin was made available to the public. Described by its creator, Satoshi Nakamoto, as an improved system of money, they argued that the unique technological components

Permission to make digital or hard copies of part or all of this work for personal or classroom use is granted without fee provided that copies are not made or distributed for profit or commercial advantage and that copies bear this notice and the full citation on the first page. Copyrights for third-party components of this work must be honored. For all other uses, contact the owner/author(s)

CHI'19 Extended Abstracts, May 4-9, 2019, Glasgow, Scotland UK

(1) 2019 Copyright held by the owner/author(s).

ACM ISBN 978-1-4503-5971-9/19/05.

https://doi.org/10.1145/3290607.3312969 
of Bitcoin, including the distributed ledger technology blockchain, allowed Bitcoin to be used without relying on trust [6]. Over the course of 2018, there was a large rise in the value of a Bitcoin, to approximately $\$ 19,000$ per coin, as compared to its value of a few hundred dollars prior to 2018 . This meteoric rise in price was met with massive public interest, especially for investors looking to profit from Bitcoin's rise. However, in late 2018, the market had a steady decline to about $\$ 3,800$ per coin, meaning Bitcoin lost about $80 \%$ of its value. This created a serious challenge to people who own and support Bitcoin. Many who purchased Bitcoin during the boom had lost most of their investment by late 2018 [7] and stopped using Bitcoin entirely in the face of this monetary risk.

Problems with Bitcoin have been identified as potential reasons not to have trust in its future $[1,3]$. Bitcoin depends on trust to function: trust in transactional partners, trust in the technology, and trust in Bitcoin's capacity as a shared store of value. Despite current issues, Bitcoin still has an active userbase with thousands of transactions daily and dozens of online communities praising the currency's merits. How do these users maintain their trust in Bitcoin despite current struggles? What reasoning do they use to explain and justify their continued positive outlook towards Bitcoin?

We are studying how people participating in a complex sociotechnical system (Bitcoin) work to maintain that system socially in the face of a serious existential challenge. In this paper, we characterize the work that participants in the $/ r /$ bitcoin subreddit undertake to maintain the sociotechnical system Our goal is to examine how the role of trust in maintaining Bitcoin as a sociotechnical system is influenced by current struggles. Additionally, we will be examining how a specific subset of community members justify their beliefs and articulate their group identity.

\section{Literature Review}

Bitcoin is a complex sociotechnical system; it includes a complicated distributed technology system [6], but it also relies on social structures such as mining, currency exchange, and social trust to function properly. Bitcoin has value largely because the community of practice[5] that has formed around it leads people to trust it to have value.

Interviewing Bitcoin users in the United States, Gao et al. found that Bitcoin users often misunderstand many of the technical properties of the system, but still believe that the system is an "ideal payment system", including fast transactions and increased security and privacy [2]. Particularly relevant to our work, there was some confusion about whether Bitcoin was supposed to be an investment vehicle (like a stock) or a currency that can be used for purchases (like the US Dollar). Sas and Khairudden echoed this with interviews in Malaysia, finding that many users focused on how socioeconomic properties of Bitcoin such as decentralization and unregulation impact their ability to use Bitcoin as either an investment or a currency [8]. 


\section{DATA AND ANALYSIS}

We gathered and examined comments publicly posted on the Bitcoin "r/bitcoin" subreddit forum. Our goal was to examine naturally occurring online discussions related to trust in Bitcoin and investigate the way Bitcoin trust was ingrained in everyday conversations. This forum was selected for our inquiry due to its large user base and high activity levels. Reddit Bitcoin has over one million registered subscribers, significantly higher than other major Bitcoin online communities. For instance, StackExchange Bitcoin has less than registered 100,000 users. The official Bitcoin.org forums have more registered users, but have become increasingly inactive, averaging a few hundred users online at a time while the Bitcoin reddit frequently has several thousands users online.

Our analysis took place in two stages. We began with an exploratory analysis to discover what topics and themes were prevalent on the subreddit. It was necessary to establish what topics of conversations were present in order to establish the scope and focus of our analysis. For this stage we gathered comments from a small pool of daily discussion threads. These threads reflect the diversity of topics on the subreddit. After this descriptive analysis, we focused our inquiry on identifying common patterns and themes of discussion as related to trust in Bitcoin. Trust themes present in the data were organized into a codebook, allowing us to identify patterns across a wider dataset.

For the main analysis, we examined comments posted on the Bitcoin subreddit in the span of one week in December 2018 using this codebook. During this time span, all new threads and comments were gathered every hour using the the Reddit API. Duplicate threads and comments were eliminated from the analysis. A sample of 330 threads were randomly selected from the corpus. After eliminating threads irrelevant to our codes, our final corpus consisted of 251 threads for coding and content analysis. All threads used for the main analysis were posted in the span of December 3rd - December 10th 2018. A total of 2,509 threads were collected for this time span.

\section{THE SHARED IDENTITY OF "TRUE BITCOINERS"}

In order to work toward supporting bitcoin, many participants in the community developed this shared identity that they call being a "true Bitcoiner" or simply "Bitcoiner". While the current uncertainty about Bitcoin's value has an active presence on the forum, this vocal subgroup of Bitcoiners has been actively working to share their maintained trust in Bitcoin. To maintain this shared identity, the true Bitcoiners engaged in three main activities on the subreddit. First, they utilized strategies to define their group identity and out-group individuals who do not meet this standard. Bitcoiners also redefined proposed problems with Bitcoin as inconsequential or even beneficial. Additionally, a core characteristic of themselves Bitcoiners described is loyalty, especially in terms of how their collective loyalty supports Bitcoin. 
"flowbrother: Real HODLers NEVER sell to fiat. Whether the fiat 'price' is zero or 10 million a coin, a HODLer NEVER sells." - (r/bitcoin/a2s3j8)

"trije: Still not selling, and still more bullish than a year ago. [...] To all the brave warriors here, let's aim for $2 M M$ now. HODL"(r/bitcoin/a2n8pw)

"phlogistonical:[...] You are now experiencing the price all of us are paying when we made this bet, with real skin in the game, that bitcoin is the money of the future. Imagine bitcoin ever hits $\$ 10,000,000$. Some people will think you received 'free' money and ask for part of it. You did not get it for free. You took this risk, they didn't." (r/bitcoin/a42bas)

\section{Portraying In-groups Using Language}

In many communities of practice, groups form around and are maintained by individual personal connections[5]. However, for true Bitcointers, the in-group and out-group was based more on behavioral identity and not on interpersonal relationships. There was little evidence of any personal relationships existing, forming, or being maintained on the subreddit.

Instead, a core principle of true Bitcoiners is not exchanging their Bitcoins for fiat currencies. Fiat currencies are currencies that gain their value through being backed by a government (like the US Dollar). True Bitcoiners are skeptical of Bitcoin being evaluated in terms of fiat because the market exchange rate may fluctuate in ways that do not reflect their belief in Bitcoin's value.

One way in which true Bitcoiners establish their group identity is through defining what types of behaviors define out-group members, called "non-Bitcoiners". This Bitcoiner out-grouping is primarily achieved through language use. Flowbrother's comment in the sidebar demonstrates this component of true Bitcoiner identity. True Bitcoiners use the term 'HODL' to describe holding on to their Bitcoin rather than selling to fiat. The term HODLers originated several years ago when a Bitcoiner who was describing their decision to 'hold' Bitcoin despite a price fall misspelled 'hold' as 'hodl', with the misspelling becoming popular with many Bitcoiners online [4].

'HODLers' is also a common term used by true Bitcoiners to identify themselves. Being a HODLer represents not just literally holding on to Bitcoin, but also holding on to optimism for Bitcoin's future. Further, Bitcoiners identify people not adhering to their standards as an outgroup by using derogatory language, such as calling people greedy or doubtful.

Like many true Bitcoiners on the forum, flowbrother shows skepticism towards the value of Bitcoin's fiat price, regardless of gains or loses. This suggests that the true Bitcoiner identity is closely related to not following market trends. Additionally, many comments about true Bitcoiners include terminology related to validity or authenticity, such as "real" used here. This suggests an awareness of community participants who may be identifying themselves as HODLers, but do not actually adhere to these core principles.

Trije's comment was posted in reply to a celebratory discussion for $r / b i$ tcoin reaching one million subscribers. The use of the phrase 'our plan' suggests this participant's belief that true Bitcoiners have unified, mutually understood goals for Bitcoin's future dominance. This participant suggests that as more community members begin adhering to true Bitcoiner principles, Bitcoin will become powerful enough to achieve their goal of replacing fiat currencies. 'HODL' is further used here as a type of rallying cry following this user invoking group identity language. 
"flowbrother: It's how we learn. We get in for the money. We get burned, then we look at what we bought that burnt us, we discover more than we expected. Our minds get expanded, [...] We become accumulators and HODLers and actively spread the message of the silent revolution, [...] ushering in an epoch of peace and prosperity." (r/bitcoin/a4mws8)

"vroomDotclub: [...] The trading exchange rate is a reflection on YOUR NEGATIVITY, YOUR FEAR, YOUR IGNORANCE and your lack of VALUE for a sound money system. [...] You haters just DONT COME HERE if you like a corrupt oligarchy and an ever inflationary fiat [...] When you nocoiners or instant profit seekers want to grow up and realize the truth about wallsteet and the regulators instead of labeling people who have researched it as quacks maybe then you will have something to offer." - (r/bitcoin/a4bx7a)

"MrChickyBumFunGunner: [...] Such an exciting but frustrating time. Wallets broke. Coin was lost. Doge ruled. It's was hacked, fixed, patched and driven by the community of hardcore techs, devs and people that got it. [...] Satoshi invested Bitcoin to solve the major problems of greed, corruption and basic human rights. Understand that and you won't care what the price is." (r/bitcoin/a3ahc2)

\section{Loyalty to Bitcoin}

True Bitcoiners define themselves in terms of how long they have been using Bitcoin. As phlogistonical's comment illustrates, they describe their sustained engagement with Bitcoin as the foundation of Bitcoin's success, in opposition with more fickle users.

Loyalty to Bitcoin is commonly justified in terms of resiliency and patience for Bitcoin's future. True Bitcoiner loyalty is also viewed in terms of what their collective efforts can offer Bitcoin's future success. For example, Flowbrother argues that this loyalty is a means of improving the future. They suggest that the labors of HODLers allows not only Bitcoin to succeed, but fulfill its greater purpose of improving the world.

vroomDotClub created a thread to directly blame Bitcoin's value problems on the behavior of people using it. This participant suggests that those against Bitcoin are deluded and do not accept or understand problems with the economic systems Bitcoin is designed to replace. Additionally, they out-group these non-believers though name-calling, and suggest that they should leave the subreddit entirely. This suggests that Bitcoiners believe that the behavior of Bitcoin users has a direct influence on the price.

True bitcoiners who have been using Bitcoin for an extended amount of time view their experience as a symbol of their commitment to Bitcoin. They will often recount the state of Bitcoin in the past in comparison to current uncertainty as a means to ostracize the problematic behavior of other users, such as how MrChickyBumFunGunner reminisces for the early days of Bitcoin, and identifies people chasing monetary gains as fools who have overlooked Bitcoin's potential.

\section{Dealing with problems with Bitcoin from the world}

Much of the discussion on the subreddit consisted of reacting to events happening in the world. Recently, much of this discussion was centered on problems with Bitcoin, such as a fall in price. The true Bitcoiners spent considerable effort dealing with these problems by dimishing their significance or reinterpreting them as a benefit to Bitcoin.

One issue implicated in Bitcoin's current price issues is BCash, another cryptocurrency. BCash was created as a 'branch' of Bitcoin's ledger by a group of users who wanted to make changes to the Bitcoin software. Some support migrating from Bitcoin to BCash. logical notes that BCash shows Bitcoin's ability to resist the threat BCash poses. They further suggest that those supporting BCash will face negative consequences for abandoning the beliefs of the main Bitcoin community. This suggests that this participant believes trading in solely Bitcoin as a safer alternative to using other cryptocurrencies such as BCash.

psychodelic interprets Bitcoin's price drop as a positive, due to the fact that this suggests that people are using and engaging with the currency. This participant suggests that those selling their 
"logical: Bcash was a necessary event in the history of bitcoin to demonstrate its resistance to a hard fork by the dominant player in mining. [...] Again, this is necessary to demonstrate the ineffectiveness over the long term of spinning up random coins without the right principles, community, technology, etc... Those things have a long way to fall still. Anyone trading bitcoin for shitcoins now is taking a huge risk and if they stay in that position long term they will lose everything." - (r/bitcoin/a3fyxw)

"psychodelic: To all those who say bitcoin has negative people on here. [...] It's almost like how for a dog any attention is good intention. The fact that bitcoin is dropping in price is actually good for it as an entity because that means people are selling. If people are selling that means people are involved. Sure, its dropping but people are talking about it. They're interested in it. They're intrigued by it. They're learning more. They're coming up with counter arguement. It's the topic of discussion. People at my workplace are investing it and some straight up hate it but hey. It's on their minds isnt it? That's good." (r/bitcoin/a4na0b)
Bitcoin are still remaining actively involved with the community. They believe that selling is an indicator of motivation to to form opinions about Bitcoin, an indication of personal investment. For this participant, people simply thinking about Bitcoin is positive regardless of the circumstance promoting the interest. Any attention given to Bitcoin suggests public engagement. This participant's reinterpretation of Bitcoin's price issue reflects their confidence in Bitcoin's intrigue and potential for everyday people.

\section{CONCLUSIONS}

In this study, we examined the $\mathrm{r}$ /bitcoin Reddit forum to examine how true bitcoiners justify their trust in Bitcoin. In our findings, bitcoiners justify trust by in-grouping themselves using language, defining their group identity in terms of loyalty, and redefining the significance of potential problems. Bitcoiners believe that Bitcoin needs dedicated social support now in order to expand and eventually have wide social impacts. This inquiry has implications for understanding how participants in a socio-technical system use online communities to develop understanding of the system and define their identity within it.

\section{ACKNOWLEDGMENTS}

This material is based upon work supported by the National Science Foundation under Grant No. 1350253 (https://www.nsf.gov/awardsearch/showAward?AWD_ID=1350253).

\section{REFERENCES}

[1] M. Conti, E. Sandeep Kumar, C. Lal, and S. Ruj. 2018. A Survey on Security and Privacy Issues of Bitcoin. IEEE Communications Surveys Tutorials 20, 4 (Fourthquarter 2018), 3416-3452. https://doi.org/10.1109/COMST.2018.2842460

[2] Xianyi Gao, Gradeigh D. Clark, and Janne Lindqvist. 2016. Of Two Minds, Multiple Addresses, and One Ledger: Characterizing Opinions, Knowledge, and Perceptions of Bitcoin Across Users and Non-Users. In Proceedings of the 2016 CHI Conference on Human Factors in Computing Systems (CHI '16). ACM, New York, NY, USA, 1656-1668. https: //doi.org/10.1145/2858036.2858049

[3] Florian Hawlitschek, Benedikt Notheisen, and Timm Teubner. 2018. The limits of trust-free systems: A literature review on blockchain technology and trust in the sharing economy. Electronic Commerce Research and Applications 29 (2018), 50 - 63. https://doi.org/10.1016/j.elerap.2018.03.005

[4] Shawn Langlois and Jessica Marmor Shaw. 2018. 'Hodling' bitcoin: The term is so popular, it made it into Senate testimony. MarketWatch (February 6 2018).

[5] Jean Lave and Etienne Wenger. 1991. Situated Learning: Legitimate Peripheral Participation. Cambridge University Press.

[6] Satoshi Nakamoto. 2008. Bitcoin: A Peer-to-Peer Electronic Cash System. Unpublished (2008).

[7] Nathaniel Popper and Su-Hyun Lee. 2018. After the Bitcoin Boom: Hard Lessons for Cryptocurrency Investors. New York Times (August 20 2018).

[8] Corina Sas and Irni Eliana Khairuddin. 2017. Design for Trust: An Exploration of the Challenges and Opportunities of Bitcoin Users. In Proceedings of the 2017 CHI Conference on Human Factors in Computing Systems (CHI '17). ACM, New York, NY, USA, 6499-6510. https://doi.org/10.1145/3025453.3025886 